\title{
Multiplicative Reverse Geometric-Arithmetic Indices and Arithmetic-Geometric of Silicate Network
}

\author{
Gowtham Kalkere Jayanna ${ }^{1, *} \mathbb{D}$, Narahari Narasimha Swamy ${ }^{1}$ \\ 1 Dept.of Mathematics, University College of Science, Tumkur University,Tumakuru, Karnataka State, Pin 572 103, India \\ * Correspondence: gowtham_k_j@yahoo.com (G.K.J.);
}

Received: 14.05.2021; Revised: 28.06.2021; Accepted: 1.07.2021; Published: 13.08.2021

\begin{abstract}
In mathematical chemistry, geometric-arithmetic and arithmetic-geometric indices are helpful from both theoretical and practical points of view. We defined two new topological indices, multiplicative reverse geometric-arithmetic and arithmetic-geometric indices, and found the same for some silicate networks.
\end{abstract}

Keywords: silicate network; reverse vertex degree; multiplicative reverse geometric-arithmetic index; multiplicative reverse arithmetic-geometric index;

(C) 2021 by the authors. This article is an open-access article distributed under the terms and conditions of the Creative Commons Attribution (CC BY) license (https://creativecommons.org/licenses/by/4.0/).

\section{Introduction}

Let $G=(V, E)$ be a simple graph with a vertex set $V=V(G)$ and an edge set $E=$ $E(G)$.. The degree of a vertex $v_{i}$ is the number of edges attached with it and denoted by $d_{G}\left(v_{i}\right)$. Let $\Delta(G)$ be the maximum degree of vertex among the vertices of a graph $G$. In [1], reverse vertex degree $c\left(v_{i}\right)$ of the vertex $v_{i}$ is defined as $c\left(v_{i}\right)=\Delta(G)-d_{G}\left(v_{i}\right)+1$.

Topological indices collect information from the molecule's graph and help predict the properties of the underlined molecule. A molecular or chemical graph is a simple graph in which vertices and edges represent atoms and bonds. Topological indices collect information from the graph of the molecule and help predict an underlined molecule's properties. Various topological indices have been considered in theoretical chemistry and have found applications, especially in QSPR/QSAR research.

In [2], the first multiplicative arithmetic-geometric index of a graph $G$ is defined as follows:

$$
\operatorname{AG\Pi }(G)=\prod_{u v \in E(G)} \frac{d_{G}(\mathrm{u})+d_{G}(v)}{2 \sqrt{d_{G}(\mathrm{u}) \cdot d_{G}(\mathrm{v})}}
$$

In [3], the first multiplicative geometric-arithmetic index of a graph $G$ is defined as follows:

$$
G A \Pi(G)=\prod_{u v \in E(G)} \frac{2 \sqrt{d_{G}(\mathrm{u}) \cdot d_{G}(\mathrm{v})}}{d_{G}(\mathrm{u})+d_{G}(\mathrm{v})}
$$

Recently using reverse vertex degrees, new topological indexes are found in [4-6]. 
Recently, in [7], it is found that the arithmetic-geometric index is useful from a practical point of view. To know extremal graphs for each bound involving this index allows for detecting chemical compounds that could satisfy desirable properties. Hence, these extremal graphs should correspond to molecules with an extremal value of the desired property correlated well with this index.

The applications of both arithmetic-geometric index and geometric-arithmetic index are found in the literature [8-11]

Motivated by applications of the arithmetic-geometric index and geometric-arithmetic index, we in this paper define the multiplicative reverse geometric-arithmetic index and multiplicative reverse arithmetic-geometric index as follows:

The multiplicative reverse arithmetic-geometric index of a graph $G$ is defined as follows:

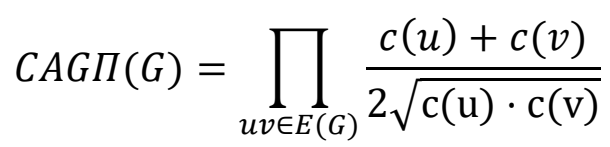

The multiplicative reverse geometric-arithmetic index of a graph $G$ is defined as follows:

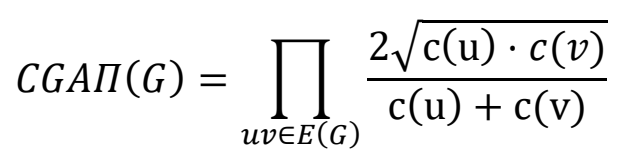

In section 3, we aim to compute multiplicative reverse arithmetic-geometric index and multiplicative reverse geometric-arithmetic index for Rhombus silicate network, $S i_{2} C_{3} I[r, s]$, and $\mathrm{Si}_{2} \mathrm{C}_{3} I I[r, s]$.

\section{Materials and Methods}

\subsection{Rhombus silicate network.}

Consider an $n$ dimensional honeycomb network $H C(n)$ and place silicate ions on all the vertices of $H C(n)$. Now subdivide each edge of it one time and place oxygen ions on the new vertices formed. Set up $6 n$ new pendant edges at 2-degree silicon ions of $H C(n)$ and place oxygen ions at the pendant vertices. Each silicon ion connects the three adjacent oxygen ions to form a tetrahedron. The resulting network is silicate network $S L(n)$.

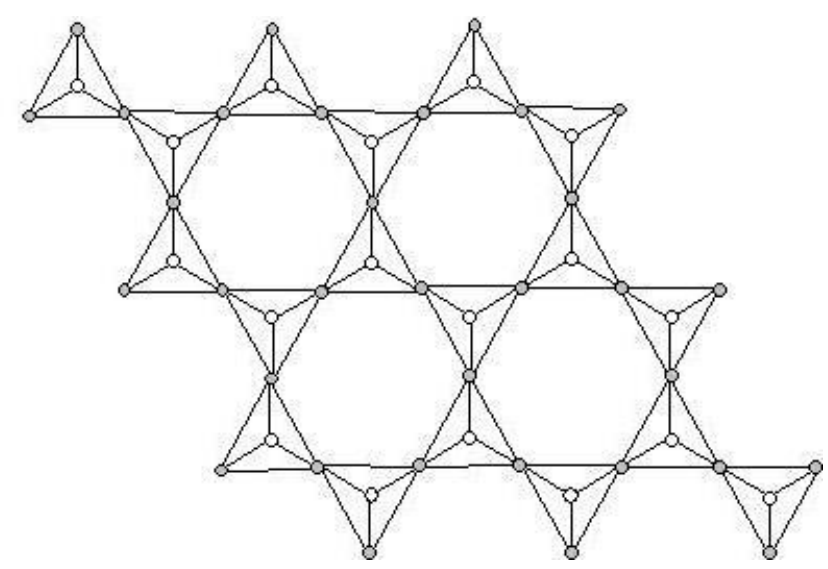

Figure 1. Rhombus silicate network $\left(R H S L_{n}\right)$. 
2.2. Silicon carbide $\mathrm{Si}_{2} \mathrm{C}_{3} I[r, s]$ and $\mathrm{Si}_{2} C_{3} I I[r, s]$.

In 1891, an American scientist discovered silicon carbide. Nowadays, we can produce silicon carbide artificially by silica and carbon[16]. Up to 1929, the hardest material was silicon carbide on earth, and Mohs hardness rating is 9, which was found similar to diamond.

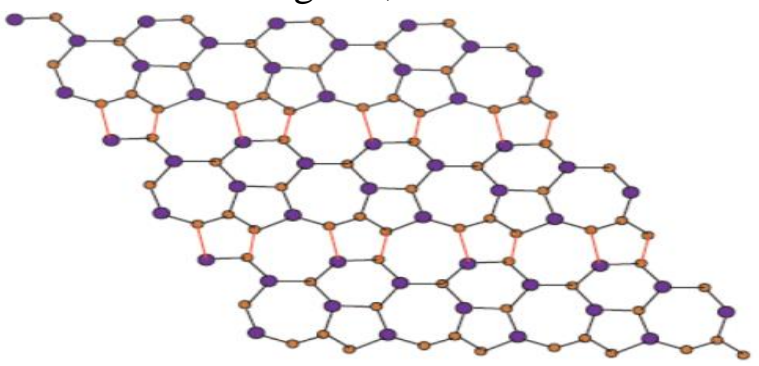

Figure 2. $S i_{2} C_{3} I[r, s]$ for $r=4, s=2$.

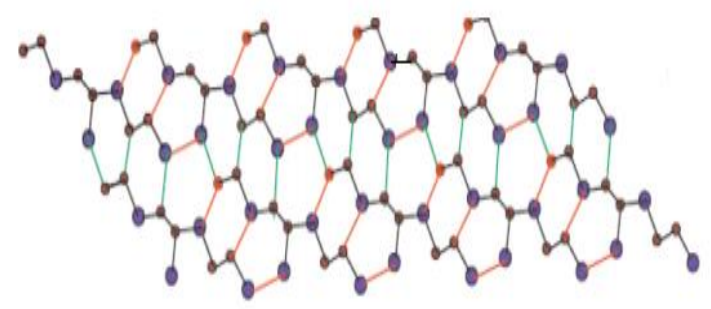

Figure 3. $\mathrm{Si}_{2} C_{3} I I[r, s]$ forr $=5, s=2$.

\section{Results and Discussion}

\subsection{Result for rhombus silicate network.}

Theorem 3.1.1.

The reverse multiplicative geometric-arithmetic index of rhombus silicate network $R H S L_{n}$ is: $C \mathrm{GA} \Pi\left(R H S L_{n}\right)=\left(\frac{4}{5}\right)^{6 n^{2}+4 n-4}$

Proof:

In $R H S L_{n}$ network has $5 n^{2}+2 n$ vertices and $12 n^{2}$ edges. Clearly, the vertices of $R H S L_{n}$ are either of degrees 3 or 6 . Then the maximum degree of a vertex, $\triangle\left(R H S L_{n}\right)=6$.

In $R H S L_{n}$, by algebraic method, there are three types of edge partitions as follows,

$$
\begin{gathered}
E_{\{3,3\}}\left(R H S L_{n}\right)=\left\{e=\left.u v \in E\left(R H S L_{n}\right)\right|_{\left.d_{R H S L_{n}}(u)=3=d_{R H S L_{n}}(v)\right\},}\right. \\
E_{\{3,6\}}\left(R H S L_{n}\right)=\left\{e=\left.u v \in E\left(R H S L_{n}\right)\right|_{d_{R H S L_{n}}(u)=3, d_{R H S L_{n}}(v)=6}\right\} \text { and } \\
E_{\{6,6\}}\left(R H S L_{n}\right)=\left\{e=\left.u v \in E\left(R H S L_{n}\right)\right|_{\left.d_{R H S L_{n}}(u)=6=d_{R H S L_{n}}(v)\right\} \text { suchthat, }}\right. \\
\left|E_{\{3,3\}}\left(R H S L_{n}\right)\right|=4 n+2,\left|E_{\{3,6\}}\left(R H S L_{n}\right)\right|=6 n^{2}+4 n-4 \text { and } \\
\left|E_{\{6,6\}}\left(R H S L_{n}\right)\right|=6 n^{2}-8 n+2 \text { We have reverse vertex degree, } \\
c_{v}=\Delta(G)-d_{G}(v)+1=7-d_{G}(v) \\
\text { Now we see reverse edge partitions as follows: }
\end{gathered}
$$

$C E_{\{4,4\}}\left(R H S L_{n}\right)=\left\{e=\left.u v \in E\left(R H S L_{n}\right)\right|_{c_{u}=4=c_{v}}\right\}$,

$C E_{\{4,1\}}\left(R H S L_{n}\right)=\left\{e=\left.u v \in E\left(R H S L_{n}\right)\right|_{c_{u}=4, c_{v}=1}\right\}$ and

$C E_{\{1,1\}}\left(R H S L_{n}\right)=\left\{e=\left.u v \in E\left(R H S L_{n}\right)\right|_{c_{u}=1=c_{v}}\right\}$. 


\begin{tabular}{|c|c|}
\hline $\begin{array}{l}\boldsymbol{c}_{\boldsymbol{u}}, \boldsymbol{c}_{\boldsymbol{v}} / \boldsymbol{u v} \\
\in \boldsymbol{E}\left(\boldsymbol{R} \boldsymbol{H} \boldsymbol{S} \boldsymbol{L}_{\boldsymbol{n}}\right)\end{array}$ & $\begin{array}{c}\text { Number of } \\
\text { reverse edges }\end{array}$ \\
\hline$(4,4)$ & $4 n+2$ \\
\hline$(4,1)$ & $6 n^{2}+4 n-4$ \\
\hline$(1,1)$ & $6 n^{2}-8 n+2$ \\
\hline
\end{tabular}

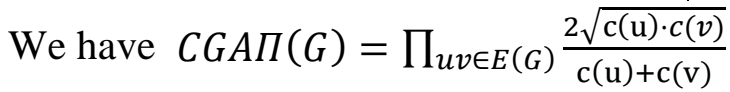

By using Table 1, we have

$$
\begin{aligned}
\operatorname{CGA\Pi }\left(\operatorname{RHSL}_{n}\right) & =\left(\frac{2 \sqrt{4 \cdot 4}}{4+4}\right)^{4 n+2} \times\left(\frac{2 \sqrt{4 \cdot 1}}{4+1}\right)^{6 n^{2}+4 n-4} \times\left(\frac{2 \sqrt{1 \cdot 1}}{1+1}\right)^{6 n^{2}-8 n+2} \\
& =\left(\frac{4}{5}\right)^{6 n^{2}+4 n-4}
\end{aligned}
$$

Theorem 3.1.2.

The reverse multiplicative arithmetic-geometric index of rhombus silicate networkRHSL $L_{n}$ is: $C A G \Pi\left(R H S L_{n}\right)=\left(\frac{5}{4}\right)^{6 n^{2}+4 n-4}$

Proof:

We have, $C A G \Pi(G)=\prod_{u v \in E(G)} \frac{c(u)+c(v)}{2 \sqrt{\mathrm{c}(\mathrm{u}) \cdot \mathrm{c}(\mathrm{v})}}$ by using Table 1, we have:

$$
\begin{aligned}
\operatorname{CAG\Pi }\left(\operatorname{RHSL}_{n}\right) & =\left(\frac{4+4}{2 \sqrt{4 \cdot 4}}\right)^{4 n+2} \times\left(\frac{4+1}{2 \sqrt{4 \cdot 1}}\right)^{6 n^{2}+4 n-4} \times\left(\frac{1+1}{2 \sqrt{1 \cdot 1}}\right)^{6 n^{2}-8 n+2} \\
& =\left(\frac{5}{4}\right)^{6 n^{2}+4 n-4}
\end{aligned}
$$

3.2.Result for silicon carbide $\mathrm{Si}_{2} C_{3} I[r, s]$.

Theorem 3.2.1.

The reverse multiplicative geometric-arithmetic index of $S i_{2} C_{3} I[r, s]$ is $C \mathrm{GA} \Pi\left(S i_{2} C_{3} I[r, s]\right)=\left(\frac{2 \sqrt{6}}{5}\right) \times\left(\frac{\sqrt{3}}{2}\right) \times\left(\frac{2 \sqrt{2}}{3}\right)^{6 r+8 s-9}$

Proof:

In $S i_{2} C_{3} I[r, s]$ have10rs vertices and $15 r s-2 r-3 s$ edges. There are five edge partitions in $S i_{2} C_{3} I[r, s]$ and are as follows: $E_{\{1,2\}}\left(S i_{2} C_{3} I[r, s]\right)=\left\{e=\left.u v \in E\left(S i_{2} C_{3} I[r, s]\right)\right|_{d_{S i_{2} C_{3} I[r, s]}(u)=1, d_{S i_{2} C_{3} I[r, s]}(v)=2}\right\}$,

$E_{\{1,3\}}\left(S i_{2} C_{3} I[r, s]\right)=\left\{e=\left.u v \in E\left(S i_{2} C_{3} I[r, s]\right)\right|_{d_{S i_{2} C_{3} I[r, s]}(u)=1, d_{S i_{2} C_{3} I[r, s]}(v)=3}\right\}$,

$E_{\{2,2\}}\left(S i_{2} C_{3} I[r, s]\right)=\left\{e=\left.u v \in E\left(S i_{2} C_{3} I[r, s]\right)\right|_{d_{S i_{2} C_{3} I[r, s]}(u)=2, d_{S i_{2} C_{3} I[r, s]}(v)=2}\right\}$,

$E_{\{2,3\}}\left(S i_{2} C_{3} I[r, s]\right)=\left\{e=\left.u v \in E\left(S i_{2} C_{3} I[r, s]\right)\right|_{d_{S i_{2} C_{3} I[r, s]}(u)=2, d_{S i_{2} C_{3} I[r, s]}(v)=3}\right\} \quad$ and $E_{\{3,3\}}\left(S i_{2} C_{3} I[r, s]\right)=\left\{e=\left.u v \in E\left(S i_{2} C_{3} I[r, s]\right)\right|_{d_{S i_{2} C_{3} I[r, s]}(u)=3, d_{S i_{2} C_{3} I[r, s]}(v)=3}\right\}, \quad$ such that $\left|E_{\{1,2\}}\left(S i_{2} C_{3} I[r, s]\right)\right|=1$,

$\left|E_{\{1,3\}}\left(S i_{2} C_{3} I[r, s]\right)\right|=1$,

$\left|E_{\{2,2\}}\left(S i_{2} C_{3} I[r, s]\right)\right|=r+2 s$, 
$\left|E_{\{2,3\}}\left(S i_{2} C_{3} I[r, s]\right)\right|=6 r+8 s-9$,

$\left|E_{\{3,3\}}\left(S i_{2} C_{3} I[r, s]\right)\right|=15 r s-9 r-13 s+7$.

As, the maximum degree in $S i_{2} C_{3} I[r, s]$ is 3 so, $c_{v}=\Delta(G)-d_{G}(v)+1=4-$ $d_{G}(v)$.The reverse edge partition is as follows:

$C E_{\{3,2\}}\left(S i_{2} C_{3} I[r, s]\right)=\left\{e=\left.u v \in\left(S i_{2} C_{3} I[r, s]\right)\right|_{c_{u}=3, c_{v}=2}\right\}$,

$C E_{\{3,1\}}\left(S i_{2} C_{3} I[r, s]\right)=\left\{e=\left.u v \in E\left(S i_{2} C_{3} I[r, s]\right)\right|_{c_{u}=3, c_{v}=1}\right\}$,

$C E_{\{2,2\}}\left(S i_{2} C_{3} I[r, s]\right)=\left\{e=\left.u v \in E\left(S i_{2} C_{3} I[r, s]\right)\right|_{c_{u}=2, c_{v}=2}\right\}$,

$C E_{\{2,1\}}\left(S i_{2} C_{3} I[r, s]\right)=\left\{e=\left.u v \in E\left(S i_{2} C_{3} I[r, s]\right)\right|_{c_{u}=2, c_{v}=1}\right\}$ and

$C E_{\{1,1\}}\left(S i_{2} C_{3} I[r, s]\right)=\left\{e=\left.u v \in E\left(S i_{2} C_{3} I[r, s]\right)\right|_{c_{u}=1, c_{v}=1}\right\}$.

Table 2. Reverse edge partition of $S i_{2} C_{3} I[r, s]$.

\begin{tabular}{c|c}
$\begin{array}{c}\boldsymbol{c}_{\boldsymbol{u}}, \boldsymbol{c}_{\boldsymbol{v}} / \boldsymbol{u} \boldsymbol{v} \\
\in \boldsymbol{E}\left(\boldsymbol{S} \boldsymbol{i}_{\mathbf{2}} \boldsymbol{C}_{\mathbf{3}} \boldsymbol{I}[\boldsymbol{r}, \boldsymbol{s}]\right)\end{array}$ & $\begin{array}{c}\text { Number of } \\
\text { reverse edges }\end{array}$ \\
\hline$(3,2)$ & 1 \\
\hline$(3,1)$ & 1 \\
\hline$(2,2)$ & $r+2 s$ \\
\hline$(2,1)$ & $6 r+2 s-9$ \\
\hline$(1,1)$ & $\begin{array}{l}15 r s-13 r-13 s \\
+7\end{array}$
\end{tabular}

We have,

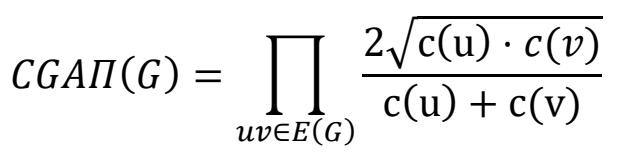

By using Table 2, we have

$$
\begin{aligned}
& \operatorname{CGA\Pi }\left(S i_{2} C_{3} I[r, s]\right)=\left(\frac{2 \sqrt{3 \cdot 2}}{3+2}\right)^{1} \times\left(\frac{2 \sqrt{3 \cdot 1}}{3+1}\right)^{1} \times\left(\frac{2 \sqrt{2 \cdot 2}}{2+2}\right)^{r+2 s} \times\left(\frac{2 \sqrt{2 \cdot 1}}{2+1}\right)^{6 r+8 s-9} \times\left(\frac{2 \sqrt{1 \cdot 1}}{1+1}\right)^{15 r s-9 r-13 s+7} \\
& =\left(\frac{2 \sqrt{6}}{5}\right) \times\left(\frac{\sqrt{3}}{2}\right) \times\left(\frac{2 \sqrt{2}}{3}\right)^{6 r+8 s-9}
\end{aligned}
$$

Theorem 3.2.2.

The reverse multiplicative arithmetic-geometric indexof $S i_{2} C_{3} I[r, s]$ is $C A G \Pi\left(S i_{2} C_{3} I[r, s]\right)=\left(\frac{5}{2 \sqrt{6}}\right) \times\left(\frac{2}{\sqrt{3}}\right)^{r+2 s+1} \times\left(\frac{3}{2 \sqrt{3}}\right)^{6 r+8 s-9}$

Proof:

We have, $C A G \Pi(G)=\prod_{u v \in E(G)} \frac{c(u)+c(v)}{2 \sqrt{\mathrm{c}(\mathrm{u}) \cdot \mathrm{c}(\mathrm{v})}}$

By using Table 2, we have:

$$
\begin{aligned}
\operatorname{CAG\Pi }\left(\operatorname{Si}_{2} C_{3} I[r, s]\right) & =\left(\frac{3+2}{2 \sqrt{3 \cdot 2}}\right)^{1} \times\left(\frac{3+1}{2 \sqrt{3 \cdot 1}}\right)^{1} \times\left(\frac{2+2}{2 \sqrt{2 \cdot 2}}\right)^{r+2 s} \times\left(\frac{2+1}{2 \sqrt{2 \cdot 1}}\right)^{6 r+8 s-9} \times\left(\frac{1+1}{2 \sqrt{1 \cdot 1}}\right)^{15 r s-9 r-13 s+7} \\
& =\left(\frac{5}{2 \sqrt{6}}\right) \times\left(\frac{2}{\sqrt{3}}\right)^{r+2 s+1} \times\left(\frac{3}{2 \sqrt{3}}\right)^{6 r+8 s-9}
\end{aligned}
$$

3.3. Resultfor silicon carbide $\mathrm{Si}_{2} C_{3} I I[r, s]$.

Theorem 3.3.1.

The reverse multiplicative geometric-arithmetic index of $S i_{2} C_{3} I I[r, s]$ is $C \mathrm{GA} \Pi\left(S i_{2} C_{3} I[r, s]\right)=\left(\frac{24}{25}\right) \times\left(\frac{\sqrt{3}}{2}\right) \times\left(\frac{2 \sqrt{2}}{3}\right)^{8 r+8 s-14}$ 
Proof:

In $\mathrm{Si}_{2} C_{3} I I[r, s]$ have10rs vertices and $15 r s-2 r-3 s$ edges. There are five edge partitions in $S i_{2} C_{3} I[r, s]$ and are as follows:

$E_{\{1,2\}}\left(S i_{2} C_{3} I I[r, s]\right)=\left\{e=\left.u v \in E\left(S i_{2} C_{3} I[r, s]\right)\right|_{d_{S i_{2} C_{3} I[r, s]}(u)=1, d_{S i_{2} C_{3} I I[r, s]}(v)=2}\right\}$,

$E_{\{1,3\}}\left(S i_{2} C_{3} I[r, s]\right)=\left\{e=\left.u v \in E\left(S i_{2} C_{3} I[r, s]\right)\right|_{d_{S i_{2} C_{3} I[r, s]}(u)=1, d_{S i_{2} C_{3} I I[r, s]}(v)=3}\right\}$,

$E_{\{2,2\}}\left(S i_{2} C_{3} I[r, s]\right)=\left\{e=\left.u v \in E\left(S i_{2} C_{3} I[r, s]\right)\right|_{d_{S i_{2} C_{3} I I[r, s]}(u)=2, d_{S i_{2} C_{3} I[r, s]}(v)=2}\right\}$,

$E_{\{2,3\}}\left(S i_{2} C_{3} I[r, s]\right)=\left\{e=\left.u v \in E\left(S i_{2} C_{3} I[r, s]\right)\right|_{d_{S i_{2} C_{3} I I[r, s]}(u)=2, d_{S i_{2} C_{3} I I[r, s]}(v)=3}\right\}$

and

$E_{\{3,3\}}\left(S i_{2} C_{3} I[r, s]\right)=\left\{e=\left.u v \in E\left(S i_{2} C_{3} I I[r, s]\right)\right|_{d_{S i_{2} C_{3} I I[r, s]}(u)=3, d_{S i_{2} C_{3} I I[r, s]}(v)=3}\right\}$,such $\quad$ that

$\left|E_{\{1,2\}}\left(S i_{2} C_{3} I I[r, s]\right)\right|=1$,

$\left|E_{\{1,3\}}\left(S i_{2} C_{3} I I[r, s]\right)\right|=1$,

$\left|E_{\{2,2\}}\left(S i_{2} C_{3} I I[r, s]\right)\right|=r+2 s$,

$\left|E_{\{2,3\}}\left(S i_{2} C_{3} I I[r, s]\right)\right|=6 r+8 s-9$,

$\left|E_{\{3,3\}}\left(S i_{2} C_{3} I I[r, s]\right)\right|=15 r s-9 r-13 s+7$.

As, the maximum degree in $S i_{2} C_{3} I[r, s]$ is 3. So, $c_{v}=\Delta(G)-d_{G}(v)+1=4-d_{G}(v)$.

The reverse edge partition is as follows:

$C E_{\{3,2\}}\left(S i_{2} C_{3} I I[r, s]\right)=\left\{e=\left.u v \in\left(S i_{2} C_{3} I[r, s]\right)\right|_{c_{u}=3, c_{v}=2}\right\}$,

$C E_{\{3,1\}}\left(S i_{2} C_{3} I I[r, s]\right)=\left\{e=\left.u v \in E\left(S i_{2} C_{3} I[r, s]\right)\right|_{c_{u}=3, c_{v}=1}\right\}$,

$C E_{\{2,2\}}\left(S i_{2} C_{3} I I[r, s]\right)=\left\{e=\left.u v \in E\left(S i_{2} C_{3} I[r, s]\right)\right|_{c_{u}=2, c_{v}=2}\right\}$,

$C E_{\{2,1\}}\left(S i_{2} C_{3} I I[r, s]\right)=\left\{e=\left.u v \in E\left(S i_{2} C_{3} I[r, s]\right)\right|_{c_{u}=2, c_{v}=1}\right\}$

and

$C E_{\{1,1\}}\left(S i_{2} C_{3} I I[r, s]\right)=\left\{e=\left.u v \in E\left(S i_{2} C_{3} I[r, s]\right)\right|_{c_{u}=1, c_{v}=1}\right\}$.

Table 3. Reverse edge partition of $\mathrm{Si}_{2} C_{3} I I[r, s]$.

\begin{tabular}{c|c}
$\begin{array}{c}\boldsymbol{c}_{\boldsymbol{u}}, \boldsymbol{c}_{\boldsymbol{v}} / \boldsymbol{u} \boldsymbol{v} \\
\in \boldsymbol{E}\left(\boldsymbol{S i}_{\mathbf{2}} \boldsymbol{C}_{\mathbf{3}} \boldsymbol{I I}[\boldsymbol{r}, \boldsymbol{s}]\right)\end{array}$ & $\begin{array}{l}\text { Number of } \\
\text { reverse edges }\end{array}$ \\
\hline$(3,2)$ & 2 \\
\hline$(3,1)$ & 1 \\
\hline$(2,2)$ & $2 r+2 s$ \\
\hline$(2,1)$ & $8 r+8 s-14$ \\
\hline$(1,1)$ & $\begin{array}{l}15 r s-13 r-13 s \\
+14\end{array}$
\end{tabular}

We have,

$$
C G A \Pi(G)=\prod_{u v \in E(G)} \frac{2 \sqrt{\mathrm{c}(\mathrm{u}) \cdot c(v)}}{\mathrm{c}(\mathrm{u})+\mathrm{c}(\mathrm{v})}
$$

By using Table 3 we have,

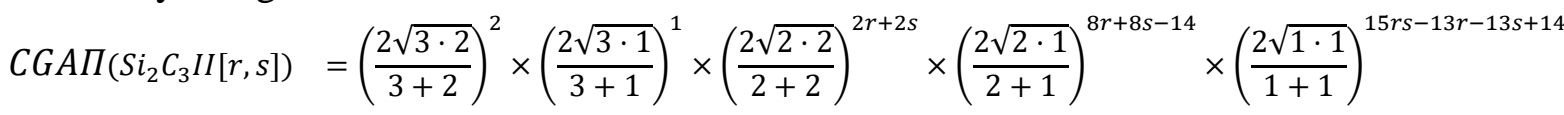

$$
\begin{aligned}
& =\left(\frac{24}{25}\right) \times\left(\frac{\sqrt{3}}{2}\right) \times\left(\frac{2 \sqrt{2}}{3}\right)
\end{aligned}
$$

Theorem 3.3.2.

The reverse multiplicative arithmetic-geometric index of $S i_{2} C_{3} I I[r, s]$ is $C A G \Pi\left(S i_{2} C_{3} I[r, s]\right)=\left(\frac{25}{24}\right) \times\left(\frac{2}{\sqrt{3}}\right) \times\left(\frac{3}{2 \sqrt{3}}\right)^{8 r+8 s-14}$

Proof: 
We have, $C A G \Pi(G)=\prod_{u v \in E(G)} \frac{c(u)+c(v)}{2 \sqrt{\mathrm{c}(\mathrm{u}) \cdot \mathrm{c}(\mathrm{v})}}$

By using Table 3, we have

$$
\begin{aligned}
\operatorname{CAG\Pi }\left(\mathrm{Si}_{2} C_{3} I I[r, s]\right) & =\left(\frac{3+2}{\sqrt{3 \cdot 2}}\right)^{2} \times\left(\frac{3+1}{\sqrt{3 \cdot 1}}\right)^{1} \times\left(\frac{2+2}{\sqrt{2 \cdot 2}}\right)^{2 r+2 s} \times\left(\frac{2+1}{\sqrt{2 \cdot 1}}\right)^{8 r+8 s-14} \times\left(\frac{1+1}{\sqrt{1 \cdot 1}}\right)^{15 r s-13 r-13 s+14} \\
& =\left(\frac{25}{24}\right) \times\left(\frac{2}{\sqrt{3}}\right) \times\left(\frac{3}{2 \sqrt{3}}\right)^{8 r+8 s-14}
\end{aligned}
$$

\section{Conclusions}

Topological indices have become a handy means for studying theoretical and practical problems in different areas of science. An imperative line of research associated with topological indices is finding optimal bounds and relations between known topological indices. The arithmetic-geometric index and geometric-arithmetic index are useful from a practical and theoretical point of view. To know extremal graphs for each bound involving this index allows detecting chemical compounds that could satisfy desirable properties. This paper defined the new reverse multiplicative arithmetic-geometric index and the reverse multiplicative geometric-arithmetic index and found the same for some silicon networks.

\section{Funding}

This research received no external funding.

\section{Acknowledgments}

The author would like to thank Dr.B.J.Gireesha, Department of mathematics, Kuvempu University, who motivated and inspired me to research. Further, I would like to thank Dr. N. Narahari, Department of mathematics, Tumkur university, for many useful suggestions.

\section{Conflict of Interest}

The authors declare no conflict of interest.

\section{References}

1. Ediz, S.; Cancan, M. Reverse Zagreb indices of Cartesian product of graphs. International Journal of Mathematics and Computer Science 2016, 11, 51-58.

2. Kulli, V.R. A new multiplicative arithmetic-geometric index. International Journal of Fuzzy Mathematical Archive 2017, 12, 49-53.

3. Kulli, V.R. Multiplicative connectivity indices of certain nanotubes. Annals of Pure and Applied Mathematics 2016, 12, 169-176,http://dx.doi.org/10.22457/apam.v12n2a8.

4. Kulli, V.R. Reverse Zagreb and reverse hyper-Zagreb indices and their polynomials of rhombus silicate networks. Annals of Pure and Applied Mathematics 2018, 16, 47-51.

5. Kulli, V.R.Computing F-reverse index and F-reverse polynomial of certain networks. International Journal of Mathematical Archive2018,9, 27-33.

6. Virk, A.U.R.; Jhangeer, M.N.; Rehman, M.A. Reverse Zagreb And Reverse Hyper-Zagreb Indices For Silicon Carbide $\mathrm{Si}_{2} \mathrm{C}_{3} I[r, s]$ and $\mathrm{Si}_{2} \mathrm{C}_{3} I I[r, s]$.Eng. Appl. Sci. Lett. 2018, 1,37-50,https://doi.org/10.30538/psrpeasl2018.0010.

7. Rodríguez, J.M.; Sánchez, J.L.; Sigarreta, J.M.; Tourís, E. Bounds on the Arithmetic-Geometric Index. Symmetry2021, 13, 689,https://doi.org/10.3390/sym13040689. 
8. Cancan, M.; Ediz, S.; Alaeiyan, M.; Farahani, M.R. On Ve-degree molecular properties of copper oxide. Journal of Information and Optimization Sciences 2020, 41, 949-957, https://doi.org/10.1080/02522667.2020.1747191.

9. Mukhtar, A.; Murtaza, R.; Rehman, S.U.; Usman, S.; Baig, A.Q. Computing the size of zero divisor graphs. Journal of Information and Optimization Sciences 2020, 41, 855-864, https://doi.org/10.1080/02522667.2020.1745378.

10. Bishan, W. Optimized model of energy industry chain considering low-carbon development mechanism. Energy Sources, Part A: Recovery, Utilization, and Environmental Effects 2020, 42, 2593-2602, https://doi.org/10.1080/15567036.2019.1612485.

11. Divnić, T.; Milivojević, M.; Pavlović, L. Extremal graphs for the geometric-arithmetic index with given minimum degree. Discrete Applied Mathematics 2014, 162, 386-390, https://doi.org/10.1016/j.dam.2013.08.001.

12. Wang, X.-L.; Liu, J.-B.; Jahanbani, A.; Siddiqui, M.K.; Rad, N.J.; Hasni, R. On Generalized Topological Indices of Silicon-Carbon. Journal of Mathematics 2020, 2128594,https://doi.org/10.1155/2020/2128594.

13. Ashrafi, A.R.;Nikzad, P. Connectivity index of the family of dendrimer nanostars, Digest Journal of Nanomaterials and Biostructures2009, 4, 269-273.

14. Kulli, V.R.; Chaluvaraju, B.; Boregowda, H.S. Some degree based connectivity indices of Kulli cycle windmill graphs. South Asian Journal of Mathematics 2016, 6, 263-268.

15. Munir, M.; Nazeer, W.; Rafique, S.; Kang, S.M. M-Polynomial and Degree-Based Topological Indices of Polyhex Nanotubes. Symmetry 2016, 8, https://doi.org/10.3390/sym8120149.

16. Gao, W.; Younas, M.; Farooq, A.; Virk, A.U.; Nazeer, W. Some Reverse Degree-Based Topological Indices and Polynomials of Dendrimers. Mathematics 2018, 6, https://doi.org/10.3390/math6100214.

17. Kulli, V.R.; Gutman, I. Computation of Sombor indices of certain networks. SSRG International Journal of Applied Chemistry 2021, 8, 1-5,https://doi.org/10.14445/23939133/IJAC-V8I1P101.

18. Kulli, V.R. Inverse sum temperature index and multiplicative inverse sum temperature index of certain nanotubes.International journal of recent scientific research2021,12, 4063540639,https://doi.org/10.24327/ijrsr.2021.1201.5734.

19. Kulli, V. Multiplicative connectivity status neighborhood indices. International journal of science engineering and technology research2020,9, 59-68,https://doi.org/10.29121/ijesrt.v9.i12.2020.8.

20. Kulli, V., Multiplicative status neighborhood indices of graphs. International journal of engineering sciences and research technology2020,9,110-120,https://doi.org/10.29121/ijesrt.v9.i6.2020.18.

21. Hosoya, H. Topological index. A newly proposed quantity characterizing the topological nature of structural isomers of saturated hydrocarbons. Bull. Chem. Soc. Jpn. 1971, 44, 2332-2339, https://doi.org/10.1246/bcsj.44.2332.

22. Asok, A.; Kureethara, J.V. QSPR Analysis of certain degree and eccentricity based topological indices and butane derivatives. Rasayan Journal of Chemistry 2019, $12, \quad 464-$ 470,http://dx.doi.org/10.31788/RJC.2019.1225093.

23. Shirakol, S.; Kalyanshetti, M.; Hosamani, S.M. QSPR analysis of certain distance based topological indices. Applied Mathematics and Nonlinear Sciences 2019, 4, 371-386, https://doi.org/10.2478/AMNS.2019.2.00032.

24. Javaid, M.; Ali, A.; Milovanović, I.; Milovanović, E. On the extremal cactus graphs for variable sum exdeg index with a fixed number of cycles. AKCE International Journal of Graphs and Combinatorics 2020, 17, 920-923, https://doi.org/10.1016/j.akcej.2019.08.007.

25. Baig, A.Q.; Naeem, M.; Gao, W.; Liu, J.-B. General fifth M-Zagreb indices and fifth M-Zagreb polynomials of carbon graphite. Eurasian chemical communications 2020, 2, 634-640, https://doi.org/10.33945/SAMI/ECC.2020.5.10.

26. Kulli, V.R. General fifth M-Zagreb indices and fifth M-Zagreb polynomials of PAMAM dendrimers. International Journal of Fuzzy Mathematical Archive 2017, 13, 99-103, https://doi.org/10.22457/ijfma.v13n1a10.

27. Asok, A.; Kureethara, J.V. QSPR Analysis of certain degree and eccentricity based topological indices and butane derivatives. Rasayan Journal of Chemistry, 2019, 12, 464-470, http://dx.doi.org/10.31788/RJC.2019.1225093.

28. Kang, S.M.; Zahid, M.A.; Nazeer, W.; Gao, W. Calculating the degree-based topological indices of dendrimers. Open Chemistry 2018, 16, 681-688, https://doi.org/10.1515/chem-2018-0071. 